\title{
Laser surface hardening of $42 \mathrm{CrMo}$ cast steel for obtaining a wide and uniform hardened layer by shaped beams
}

\author{
Peipei Sun • Shaoxia Li • Gang Yu • Xiuli He • \\ Caiyun Zheng • Weijian Ning
}

Received: 5 March 2013 / Accepted: 4 September 2013 / Published online: 29 September 2013

(C) Springer-Verlag London 2013

\begin{abstract}
For laser surface hardening (LSH) of large-sized workpieces, a wide and uniform hardened layer of a single track is pursued. In this study, two kinds of shaped laser beams were used in LSH of 42CrMo cast steel to obtain the required hardened layer. One is a stripy spot with uniform-intensity array spots and the other a stripy spot with intensity blowup in the edge of the whole array spots. As a comparison, a Gaussian laser beam was also adopted. A three-dimensional finite element model was used to simulate the thermal history of specific points by the latter shaped beam and the Gaussian laser beam. The surface morphology, microstructure, microhardness, and uniformity of hardened layers were studied. The results showed that a wider and more uniform hardened layer could be obtained using the latter shaped beam at relative higher scanning velocities and laser power. The thermal history of a material has an important effect on the microstructure and microhardness finally formed. Due to the high peak temperature and heating rate caused by the latter shaped beam, a higher value of microhardness in the transformation hardened zone was found.
\end{abstract}

Keywords Laser surface hardening (LSH) · 42CrMo cast steel $\cdot$ Laser beam shaping $\cdot$ Strip array spots

\section{Introduction}

Laser surface hardening (LSH) has been widely used in the automobile and machine tool industries due to short hardening

\footnotetext{
P. Sun $(\square)$

Basic Technology Research Center, AVIC Engine Establishment, Beijing 100028, People's Republic of China

e-mail: pipnus@yahoo.cn

$\mathrm{S} . \mathrm{Li} \cdot \mathrm{G} . \mathrm{Yu} \cdot \mathrm{X} . \mathrm{He} \cdot \mathrm{C}$. Zheng $\cdot \mathrm{W}$. Ning

Key Laboratory of Mechanics in Advanced Manufacturing, Institute of Mechanics, Chinese Academy of Sciences, Beijing 100190, People's Republic of China
}

time, small deformation after hardening, ease of automation, and selective hardening of parts [1-3]. Temperature field computation of overlapping zone or specific workpiece $[4$, 5], optimization and quantitative evaluation of the LSH process [6], and LSH by shaped beams [7-9] are the current study hot issues of LSH. Back tempering is one of the most critical problems in laser surface hardening of extended surfaces. Temper softening in overlapping zone $[5,10,11]$ will exist in multi-track LSH of large-sized workpieces such as cold roll and tug boat of rotary kiln. To solve the problem, a wide and uniform hardened layer of the single-track LSH is pursued.

To get a relatively wider hardened layer, using defocusing laser beams, increasing laser power, and decreasing the laser scanning velocity are usual methods [12]. But the most effective method is increasing laser beam size at the focal plane. Using laser beam shaping technology, a large-sized Gaussian beam [13], a wide-band beam [8], and a rectangular beam [7] were designed to get a wider single hardened layer. To get a relatively more uniform hardened layer, a customized beam with a slope intensity profile [14] and an M-shaped laser beam profile [9] were proposed and theoretically investigated. However, little literature has been reported with detailed experimental results.

In this paper, a new stripy laser beam with one-dimensional array spots produced by a self-designed diffractive optical element was used. This stripy beam is composed of a row of circular spots. According to different laser output powers, and the material properties and size of the workpieces, the number of spots and intensity distribution of the whole laser beam can be designed to get a relatively more uniform hardened layer.

As an example, two differently shaped laser beams were used in LSH of $42 \mathrm{CrMo}$ cast steel in this paper. Considering the maximum power of the used Nd:YAG laser, shaped laser beam 1 is a stripy spot with uniform-intensity array spots of 37 ; shaped laser beam 2 is a stripy spot with intensity blowup in the edge of the whole array spots which also has 37 spots. A 
Fig. 1 Schematic diagram of the optical system for laser beam shaping and application

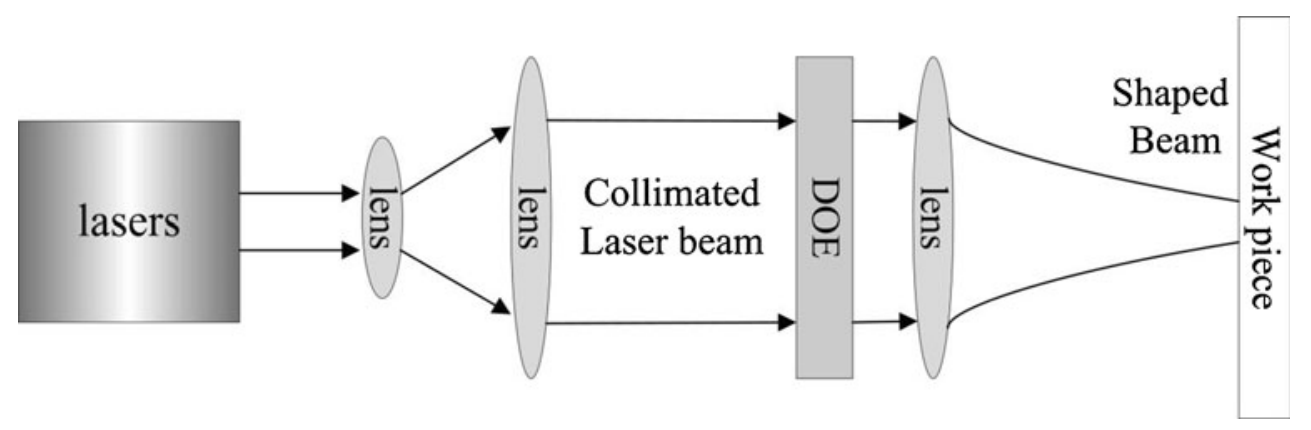

Gaussian laser beam was used as the third one. The surface morphology, microstructure, microhardness, and uniformity of the hardened layers were investigated for the three laser beams. In order to explain the results, a mathematical model of laser surface hardening of 42CrMo cast steel was established and the temperature history at specific points was calculated.

\section{Experimental procedures}

The laser source used was a fiber-coupled 1,000-W Nd:YAG laser equipped with a self-designed diffractive optical element. A schematic diagram of the optical system for laser beam shaping and application is shown in Fig. 1.
Fig. 2 Relative intensity profiles of shaped beam 1 (a) and shaped beam 2 (b)
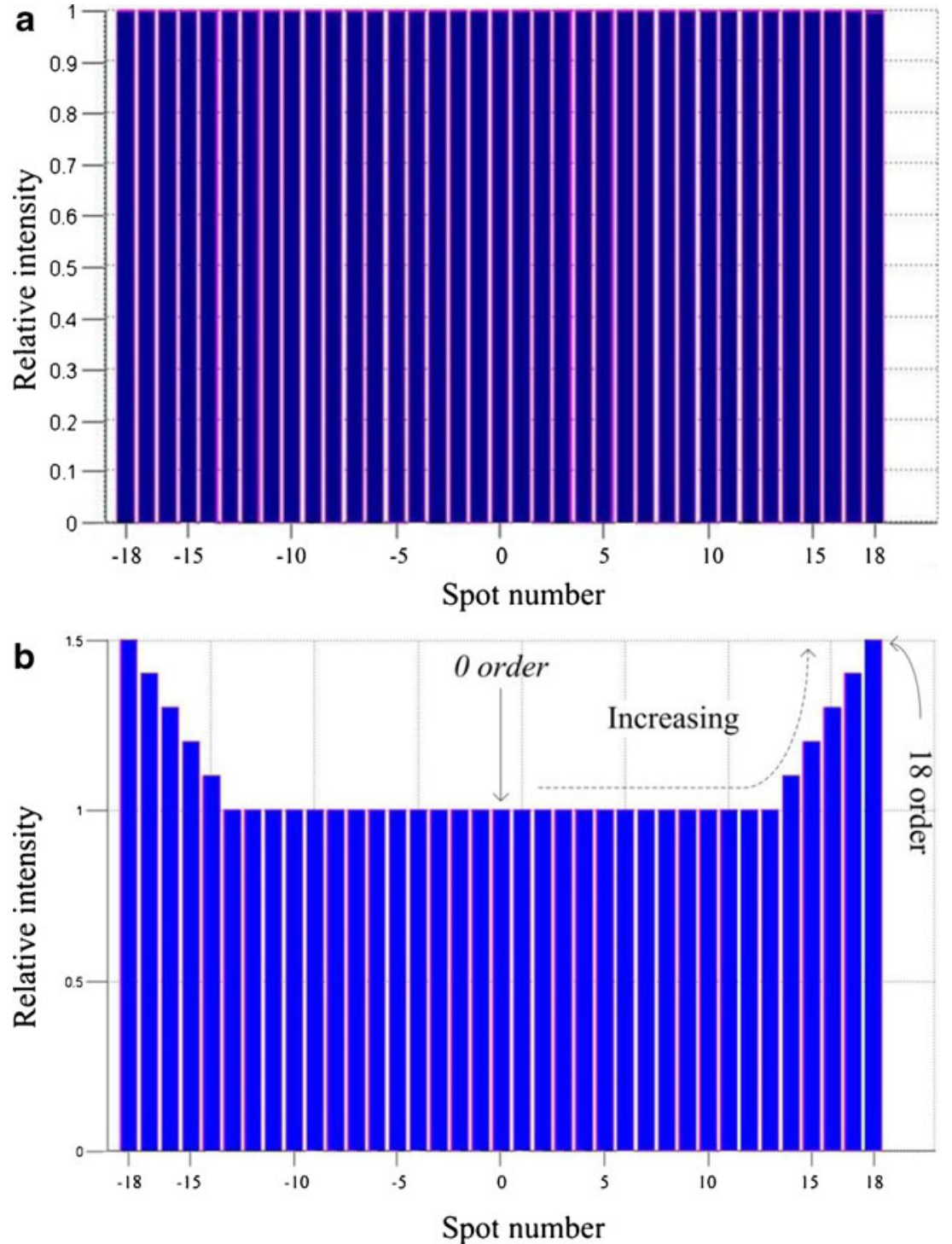
Shaped laser beams were used in this study. Shaped beam 1 is a stripy spot with uniform-intensity array spots which has 37 single-spots, as shown in Fig. 2a. The diameter of every single spot is about $0.11 \mathrm{~mm}$. Shaped beam 2 is a stripy spot with intensity blowup in the edge of the whole array spots which also has 37 single-spots, as shown in Fig. 2b. The intensity proportion of diffractive orders is:

$$
\begin{aligned}
& I_{(0)}: I_{(1)}: \ldots: I_{(13)}: I_{(14)}: I_{(15)}: I_{(16)}: I_{(17)}: I_{(18)}= \\
& 1: 1: \ldots: 1: 1.1: 1.2: 1.3: 1.4: 1.5
\end{aligned}
$$

Which means that from zero order to the 13th order, the intensity is the same, which is 1 ; from the 14th order on, the intensity grows $10 \%$ every one order.

Figure 3 shows a schematic diagram of laser surface hardening with shaped beams. Shielding gas was not used during the whole process. Two thermocouples were used to record the temperature at points $\mathrm{A}$ and $\mathrm{B}$ during the process. As shown in Fig. 3, the coordinate values of point A were: $X=$ $20 \mathrm{~mm}, Y=48 \mathrm{~mm}, Z=8 \mathrm{~mm}$; the coordinate values of point $B$ were: $X=60 \mathrm{~mm}, Y=53 \mathrm{~mm}, Z=8 \mathrm{~mm}$. The dimensions of the workpiece to be hardened were $100 \mathrm{~mm}$ (length) $\times 85 \mathrm{~mm}$ (width) $\times 8 \mathrm{~mm}$ (height).

To compare with the shaped beams, a Gaussian laser beam whose defocusing distances were from 3 to $40 \mathrm{~mm}$ was also used in the experiments. If the defocusing distance is small, a high laser power density will easily lead to vaporization for the Gaussian laser beam; when the laser power is appropriate for LSH, the hardened layer will narrow due to the small size of the Gaussian laser beam. If the defocusing distance is large enough to get a relative wider hardened layer, the output power would be high enough due to the edge energy loss of the Gaussian laser beam. The selected defocusing distance of the Gaussian laser beam is $26 \mathrm{~mm}$ to compare with the shaped beams. A laser power of $700 \mathrm{~W}$ and a laser scanning velocity of $10 \mathrm{~mm} / \mathrm{s}$ were chosen to ensure that the highest temperature obtained was just below the melting point in the case of the Gaussian laser beam.

$42 \mathrm{CrMo}$ cast steel was used in the experiment. The microstructure of the $42 \mathrm{CrMo}$ cast steel is cementite and pearlite; its nominal chemical composition (in weight percent) is $0.42 \mathrm{C}$, 0.39Si, 0.73Mn, 0.014P, 0.004S, $1.01 \mathrm{Cr}, 0.29 \mathrm{Mo}$, and balanced Fe. The fabricated sample was cross-sectioned by wireelectrode cutting, polished using standard metallographic techniques, and then chemically etched in a solution of $\mathrm{HNO}_{3}$ and $\mathrm{C}_{2} \mathrm{H}_{5} \mathrm{OH}$. The morphology and microstructure of the hardened layers were characterized with optical microscopy $(\mathrm{OM})$. Microhardness along the height centerline of the transverse section and in the longitudinal direction at $0.05 \mathrm{~mm}$ below the irradiated surface was measured by an automatic microhardness tester (HXD-1000B) using a 100-gf test force for a dwell time of $15 \mathrm{~s}$.

\section{Mathematical modeling}

In this section, a three-dimensional finite element model (FEM) was used to simulate the transient temperature field during the LSH process of the $42 \mathrm{CrMo}$ cast steel.

\subsection{Assumptions}

To simplify the calculation, the following assumptions are made:

A1. A combined heat transfer coefficient, $h_{c}$, is used to simulate the effect of radiation and convection. It can be calculated from the relation as follows:

$$
h_{\mathrm{c}}=2.41 \times 10^{-3} \varepsilon T^{1.61}
$$

where $\varepsilon$ is the emissivity and $T$ is the temperature.

A2. The thermal physical properties of the materials are temperature-dependent and piecewise linear with temperature.

\subsection{Governing equation}

The transient temperature distribution $T(x, y, z, t)$ can be calculated from the heat transfer equation with appropriate
Fig. 3 Schematic diagram of LSH with shaped beam

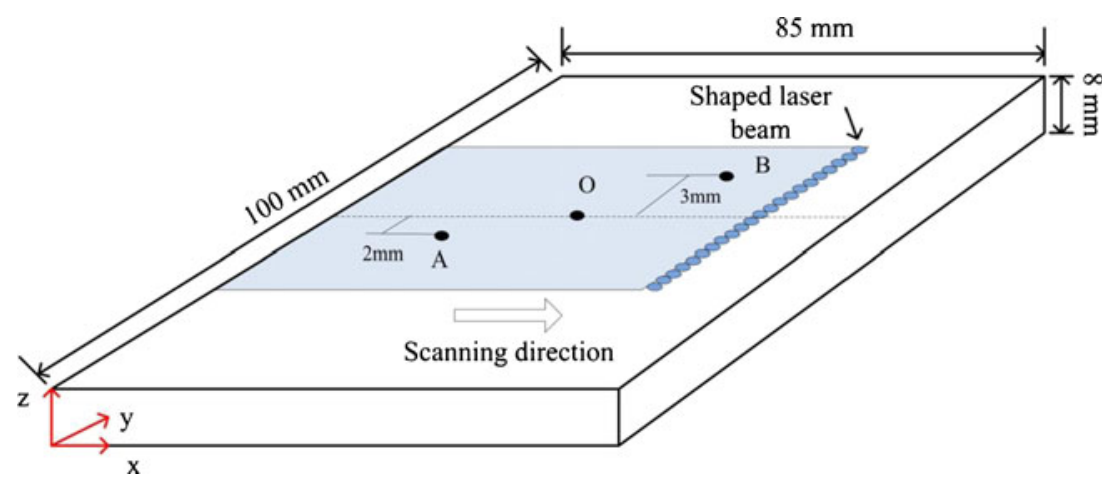




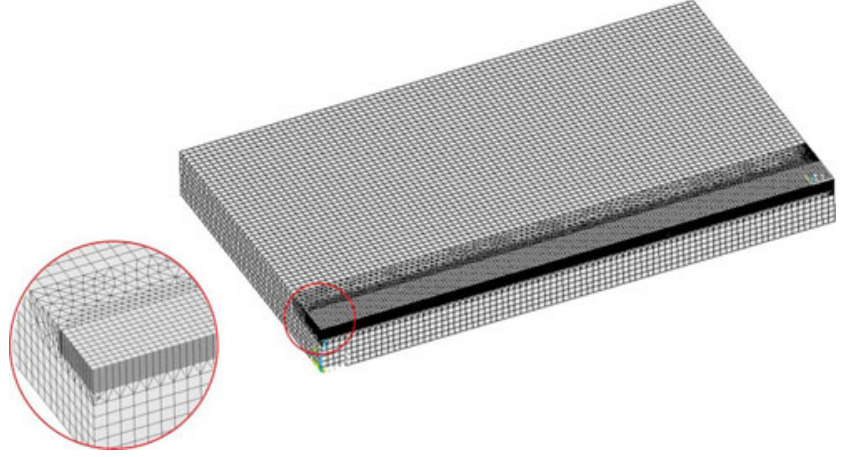

Fig. 4 Finite element mesh and geometry of the workpiece

boundary conditions. The governing equation based on the heat transfer equation is expressed as:

$\frac{\partial(\rho c T)}{\partial t}=\frac{\partial}{\partial x}\left(k \frac{\partial T}{\partial x}\right)+\frac{\partial}{\partial y}\left(k \frac{\partial T}{\partial y}\right)+\frac{\partial}{\partial z}\left(k \frac{\partial T}{\partial z}\right)$

where $\rho, c, t$ and $k$ are the density, specific heat capacity, time, and thermal conductivity, respectively.

\subsection{Boundary conditions}

The initial temperature of the materials is room temperature. Therefore, the initial condition is

$T(x, y, z, 0)=T_{\mathrm{a}}$

where $T_{a}$ is the room temperature.

The convection and radiation boundary condition can be considered together as

$k \frac{\partial T}{\partial n}=h_{\mathrm{c}}\left(T-T_{a}\right)$.

The effect of the moving laser beam can be considered as a surface heat source as follows:

$k\left(\frac{\partial T}{\partial n}\right)=a q_{m}$

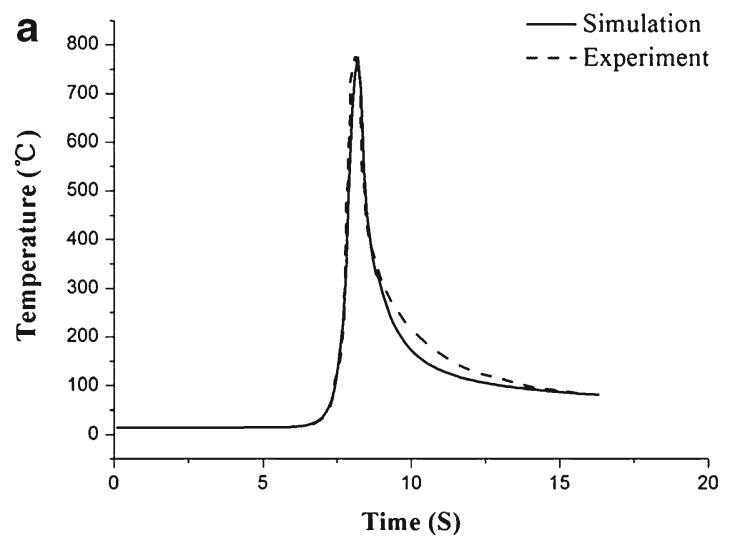

where $a$ is the absorptivity and $q_{m}$ represents the average thermal flux density.

\subsection{Numerical method}

A three-dimensional finite element model based on the above mathematical model was developed with the ANSYS parametric design language. As the geometry, boundary conditions, and the heat source were symmetric, half of the geometry was chosen for calculation. The model was shown in Fig. 4, containing three-dimensional volume elements SOLID70 with eight nodes and thermal surface effect element SURF152 defined by four nodes from the basic body and an extra node away from the base element. The threedimensional element was used for the basic body structure and the thermal surface effect element for the boundary between the structure and the environment. A denser mesh was used in the area along the laser line and a coarser mesh for the rest of the structure.

\section{Results and discussion}

\subsection{Temperature history of specific points}

Temperature readings at points A and B (Fig. 3) were used to evaluate the three-dimensional finite element model. The calculated and measured temperature history at points A and B by the Gaussian laser beam are shown in Fig. 5. The laser power and scanning velocity used were $700 \mathrm{~W}$ and $10 \mathrm{~mm} / \mathrm{s}$, respectively. It can be seen that the calculation results show a good agreement with the experimental ones.

Figure 6 shows a comparison of temperatures for shaped laser beam 2 and the Gaussian laser beam at node $O$ shown in Fig. 3, which is in the middle of the workpiece. The laser power and scanning velocity used were $700 \mathrm{~W}$ and $10 \mathrm{~mm} / \mathrm{s}$, respectively. The highest temperature in the case of the Gaussian beam is $1393{ }^{\circ} \mathrm{C}$, which is just below the melting

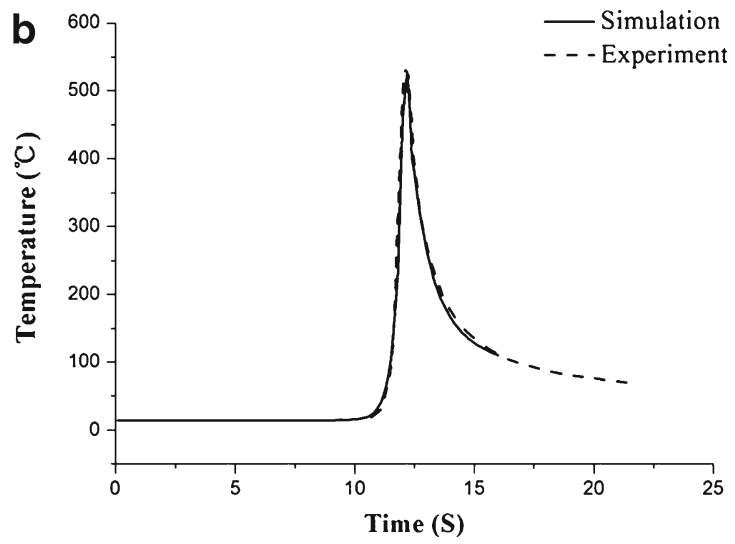

Fig. 5 Comparison between the measured and simulated temperature history at points A (a) and B (b) 


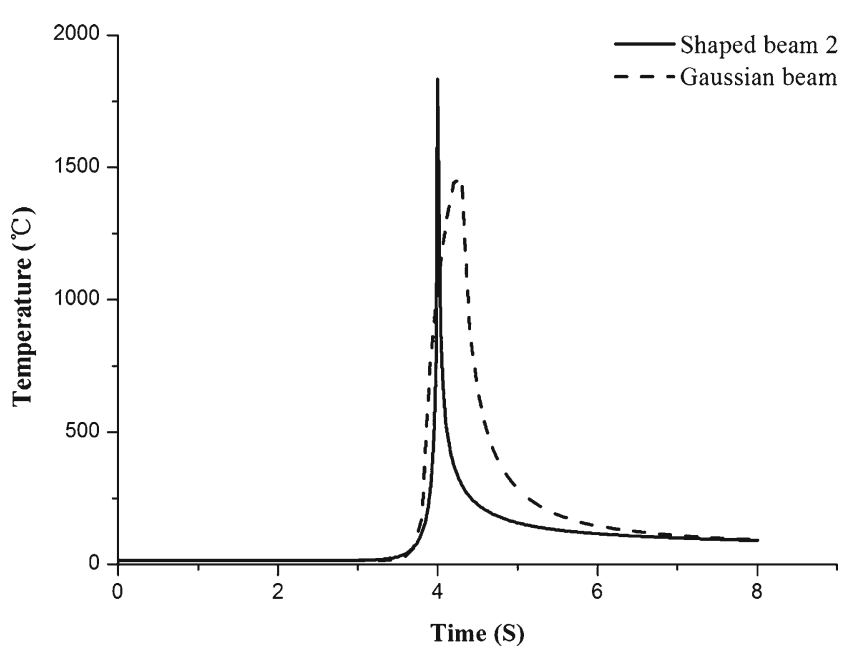

Fig. 6 Temperature versus time at $\mathrm{O}$ for shaped beam 2 and Gaussian beam

point; in the case of shaped beam 2, melting occurs. The heating rate and the peak temperature for shaped beam 2 are much higher than the Gaussian beam, and the duration of austenite transformation is much shorter. All the factors will significantly affect the microstructure and microhardness finally formed.

\subsection{Morphology}

To compare the morphology of the hardened layers by the Gaussian laser beam and the shaped laser beams, crosssections of the hardened layers using laser power of $700 \mathrm{~W}$ and scanning velocity of $10 \mathrm{~mm} / \mathrm{s}$ by the three beams are shown in Fig. 7. The width of the hardened layer is defined as the width at the top surface; the depth is defined as the maximum depth of the hardened layer. It is clear to see that the widths of the hardened layers by the shaped laser beams are much larger than that by the Gaussian beam. This means that the shaped beam has a higher energy efficiency along the width of the hardened layer due to its large length-to-width ratio.

It can be seen from Fig. 7 that the hardened layer of the sample hardened by the Gaussian laser beam is crescentshaped, while shaped beams produce a relatively flat-based or even hardened zone. Kock [15] found similar shapes of the hardened layer and explained the phenomenon as the energy absorbed per unit area across the surface perpendicular to the direction of travel of the laser beam being different. More detailed results about the uniformity will be discussed in Section 4.5. The width and depth, microhardness, and the microstructure of the hardened layers are very similar for
Fig. 7 Cross-section of the hardened layers using laser power of $700 \mathrm{~W}$ and scanning velocity of $10 \mathrm{~mm} / \mathrm{s}$ by the Gaussian beam (a), shaped beam 1 (b), and shaped beam 2 (c)

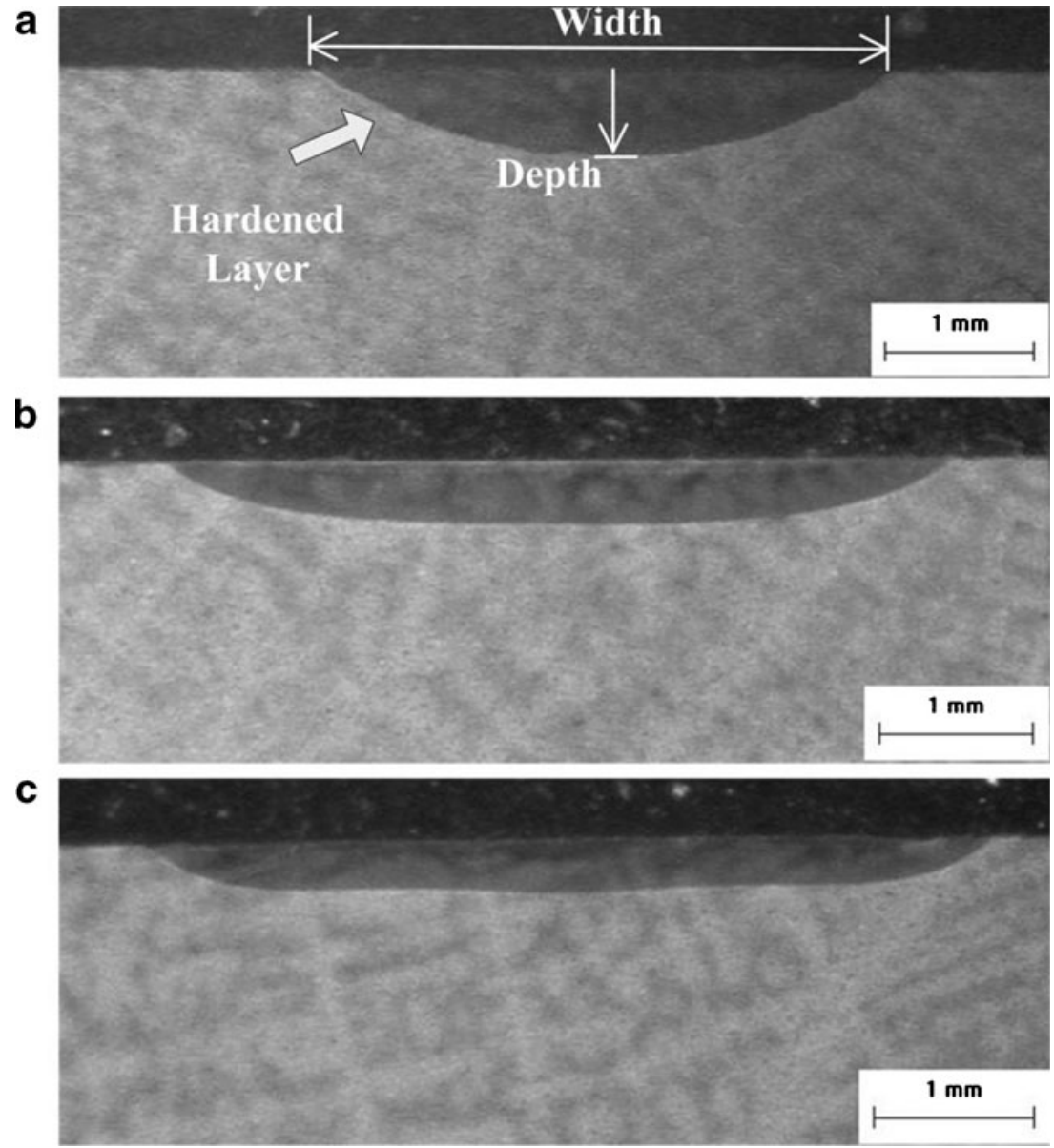



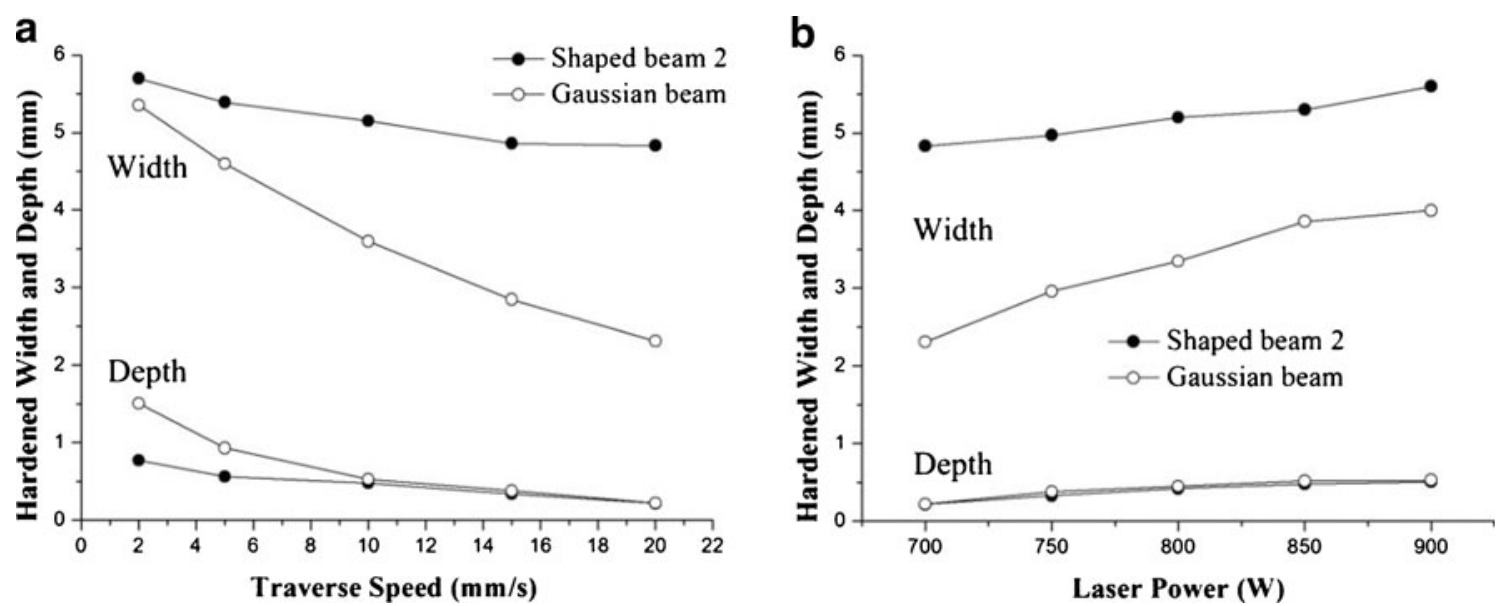

Fig. 8 Widths and depths of the hardened layers under different scanning velocities (a) and laser powers (b)

shaped beams 1 and 2, so only the comparison results of shaped beam 2 and the Gaussian beam will be given.
The widths and depths of the hardened layers under different scanning velocities (laser power, $700 \mathrm{~W}$ ) using shaped
Fig. 9 OM macrographs (a) and micrographs of the top (b), middle-upper (c), lower-middle (d), and lower (e) zones of the laser-hardened $42 \mathrm{CrMo}$ cast steel by shaped beam 2
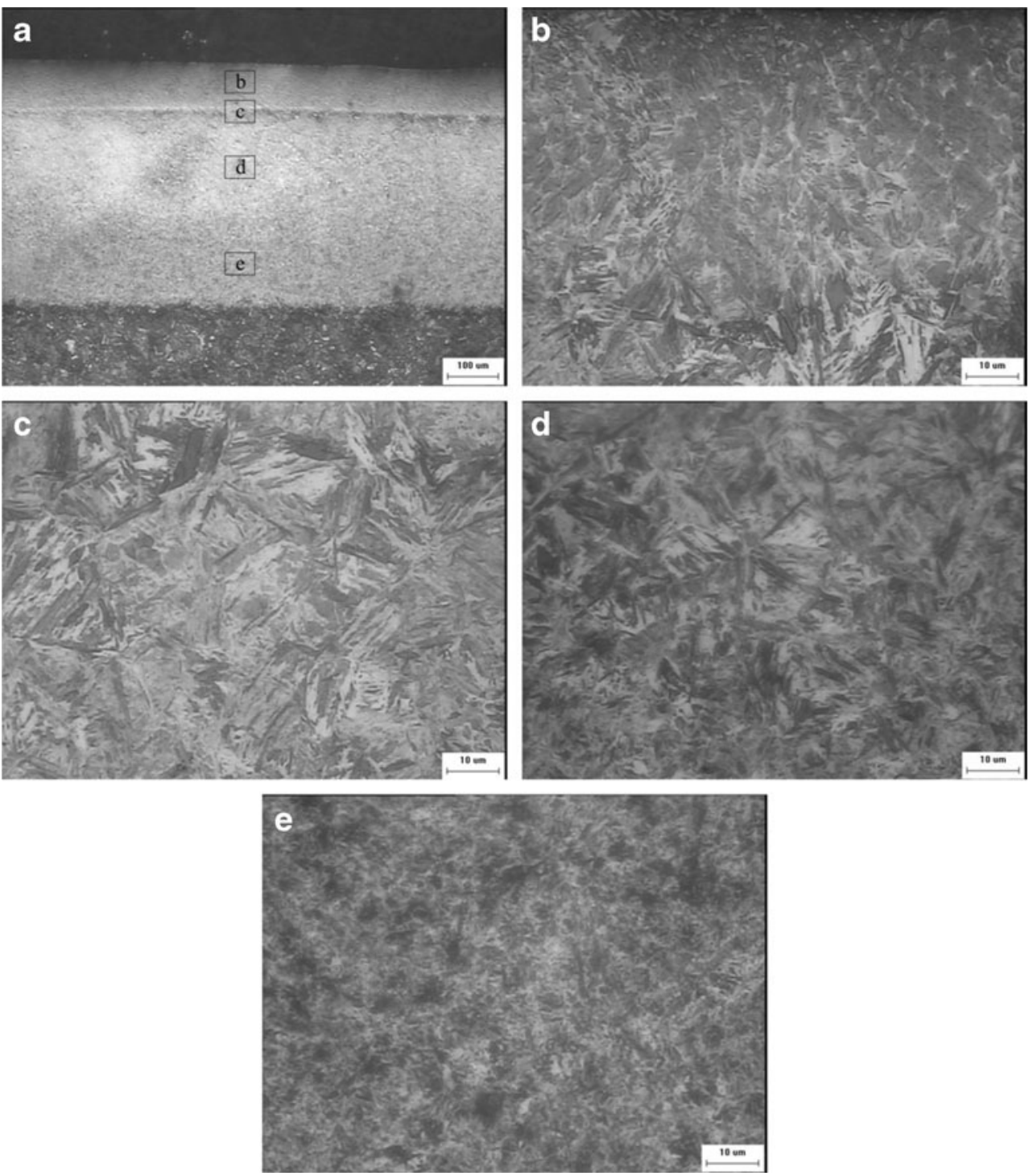
beam 2 and the Gaussian beam are shown in Fig. 8a. Figure 8b shows the widths and depths of the hardened layers under different laser powers (laser scanning velocity, $20 \mathrm{~mm} / \mathrm{s}$ ). Both Fig. 8a, b show an obvious increase in the width of the hardened layer by shaped beam 2 . The widths and depths of the layers decrease with the scanning velocity increasing for a given laser power and increase with the laser power increasing for a given scanning velocity by both beam shapes. As shown in Fig 8a, the widths of the hardened layers in the case of shaped beam 2 decrease more slowly with the scanning velocity increasing than in the case of the Gaussian laser beam, and it seems that a wider and no shallow hardened layer by the shaped laser beam can be obtained at relative higher scanning velocities. It can be seen from Fig $8 \mathrm{~b}$ that the depths are nearly the same using both laser beams when the scanning velocity is $20 \mathrm{~mm} / \mathrm{s}$.

\subsection{Microstructure}

Figure 9 shows the microstructure of the cross-section zone of the hardened layer by shaped beam 2 . It can be seen that the layer consists of a laser-melted thin surface layer of about $100 \mu \mathrm{m}$, a laser transition zone, a laser transformation hardened zone, and a heat-affected zone. In the top area of the hardened layer (Fig. 9b), butterfly martensite grows radially perpendicular to the surface of laser irradiation. In the laser transition zone (Fig. 9c), a coarse structure of lath martensite can be found, and the layer is only about $20 \mu \mathrm{m}$. This zone presents as a relative dark line in Fig. 9a.

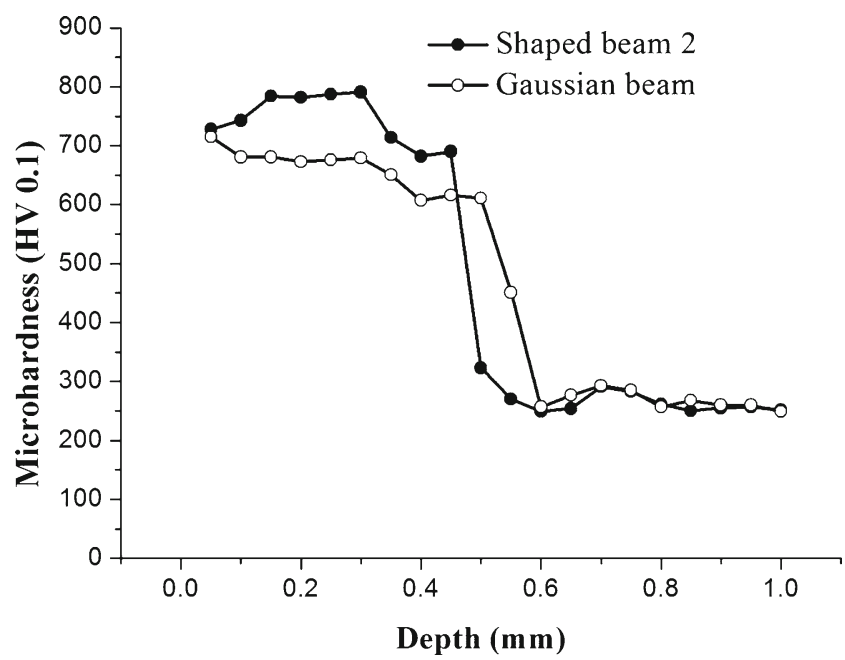

Fig. 11 Microhardness profile along the depth of the hardened layer by shaped beam 2 and the Gaussian beam

It can be seen from Fig. 9d that complete austenization has occurred following a high enough peak temperature and a rapid quenching process in the laser transformation hardened zone. A mixture of needle, lath martensite, and some areas of retained austenite can be found. In the lower region of the heat-affected zone, as shown in Fig. 9e, there are a mixed structure of tempered martensite, retained austenite, bainite, and untransformed pearlite.

The cross-section microstructure of the hardened layer by the Gaussian beam is shown in Fig. 10; the laser transformation hardened zone and the heat-affected zone can be
Fig. 10 OM macrographs (a) and micrographs of the top (b) and lower (c) zones of the laserhardened 42CrMo cast steel by the Gaussian beam
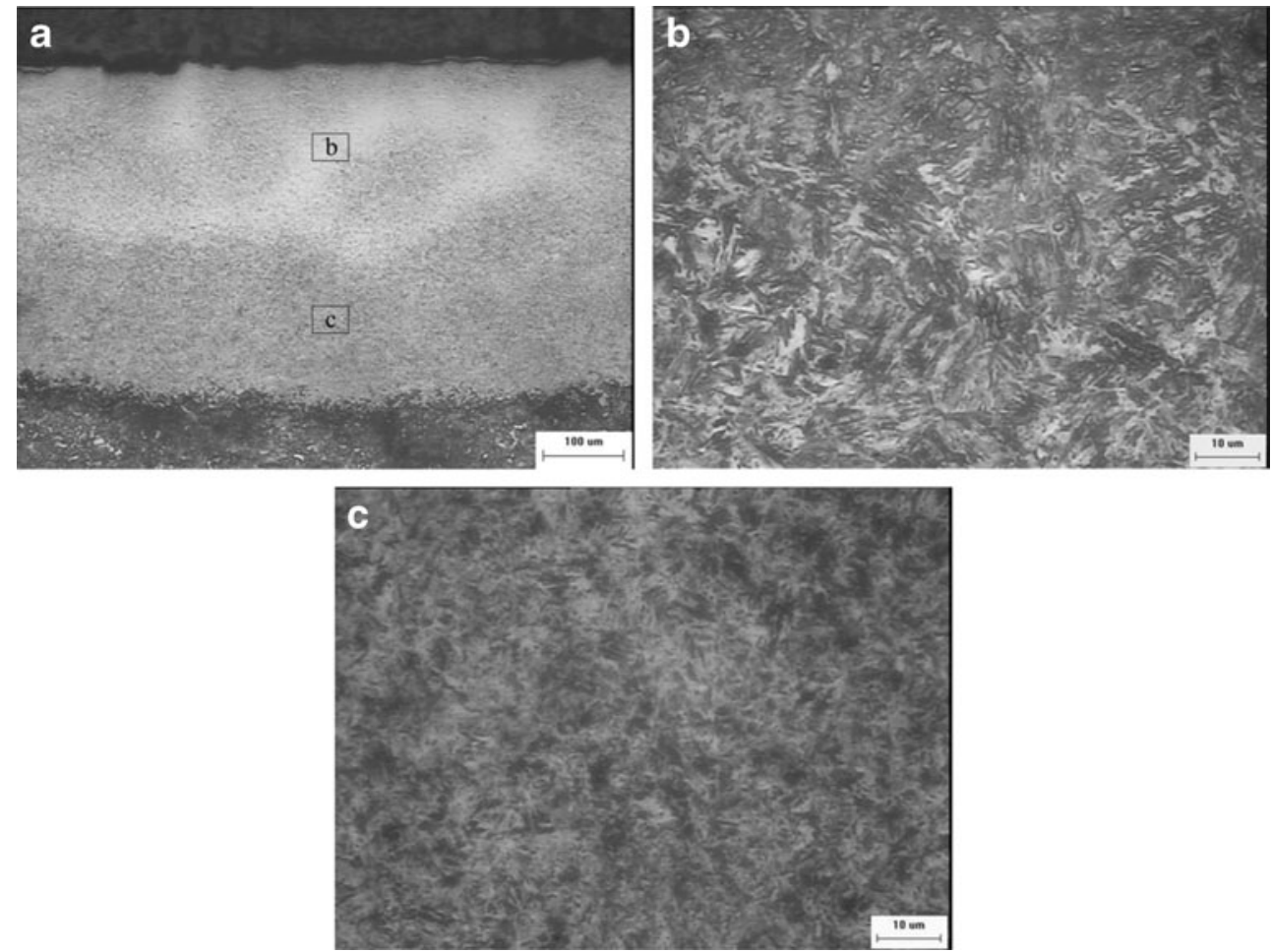


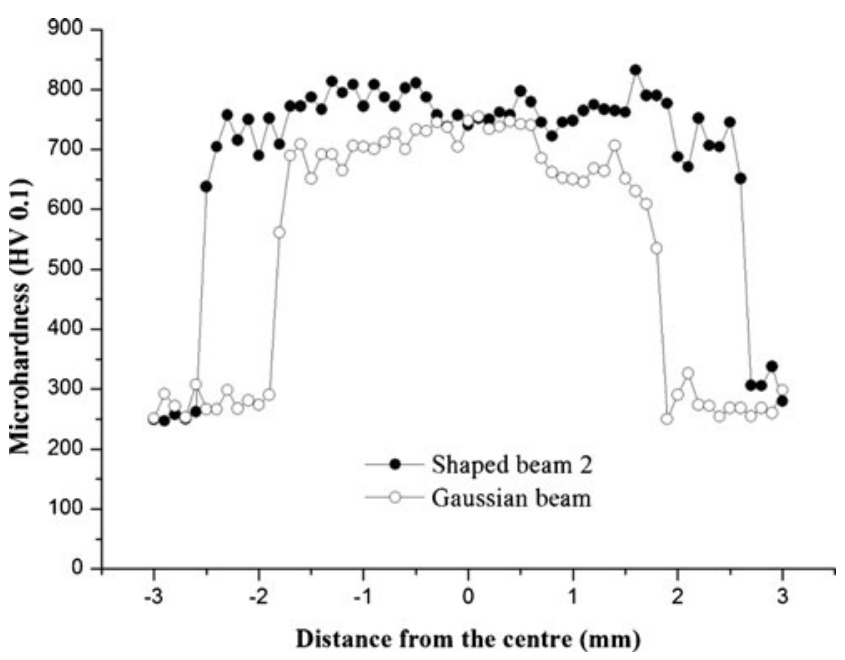

Fig. 12 Microhardness profile along the width of the hardened layer by shaped beam 2 and the Gaussian beam at $0.05 \mathrm{~mm}$ below the surface

distinguished. No melting occurs. The highest temperature is $1,393{ }^{\circ} \mathrm{C}$, which is merely below the melting point.

The top region (Fig. 10b) is exactly the same martensitic structure as that found in the hardened layer by shaped beam 2 , including needle and lath martensite. The heat-affected zone has a mixed structure of needle martensite, retained austenite, bainite, and untransformed pearlite, as shown in Fig. 10c.

\subsection{Microhardness}

Figure 11 shows the microhardness profiles along the depth of the hardened layers by shaped beam 2 and the Gaussian beam. The results confirmed the microstructure evolution discussed above. In the micro-melting zone of shaped beam 2, the hardness is about $800 \mathrm{HV} 0.1$, while in the transition zone the hardness reduces to about $730 \mathrm{HV} 0.1$ due to the appearance of coarse lath martensite. The hardness of the mixed zone is in the range of 700-750 HV 0.1, which consists of needle, lath martensite, and some areas of retained austenite. The microhardness decreases along the depth with martensite decreasing and the retained austenite increasing.
In the case of the Gaussian beam, the martensite zone hardness is $50-100 \mathrm{HV} 0.1$, lower than that in the case of shaped beam 2, besides at the upper part of the zone whose hardness is nearly the same as the transformation hardened zone by shaped beam 2. Safdar et al. [16] have reported that both the heating and cooling rates influence the outcome of hardening achieved during LSH and that higher hardness was observed with lower heating rates. Obviously, the result here is different; the possible reason is the heating rate and peak temperature both being high enough in the case of shaped beam 2, as shown in Fig. 6 . The high heating rate elevates the values of the final austenitization and homogenization temperatures, but the peak temperature is high enough, and both the high heating rate and peak temperature can accelerate the austenitization and homogenization process.

Figure 12 shows the microhardness profiles along the width of the hardened layer by shaped beam 2 and the Gaussian laser beam at $0.05 \mathrm{~mm}$ below the surface. The profiles show a uniform hardness which is about $800 \mathrm{HV}$ 0.1 in the case of shaped beam 2 and about $700 \mathrm{HV} 0.1$ in the case of the Gaussian beam. Due to the Gaussian distribution of the laser beam, hardness in the edge of the layer is a little lower than in the center. The high hardness area is narrower in the case of the Gaussian beam, which confirmed the different widths of the hardened layers by the Gaussian beam and the shaped beam well. The higher hardness which appeared in the case of shaped beam 2 is caused by the different microstructure discussed above.

\subsection{Uniformity of the hardened layer}

As shown in Fig. 13, there are two different hardened layer profiles. It is clear to see that the hardened layer profile $b$ is more uniform than profile $a$. But there is no definition of uniformity of a hardened layer, as yet. To evaluate the uniformity, a uniformity coefficient $R$ was defined, which is related as

$R=\frac{s_{1}}{s}=\frac{s_{1}}{L H}$
Fig. 13 Two different shapes of the hardened layer

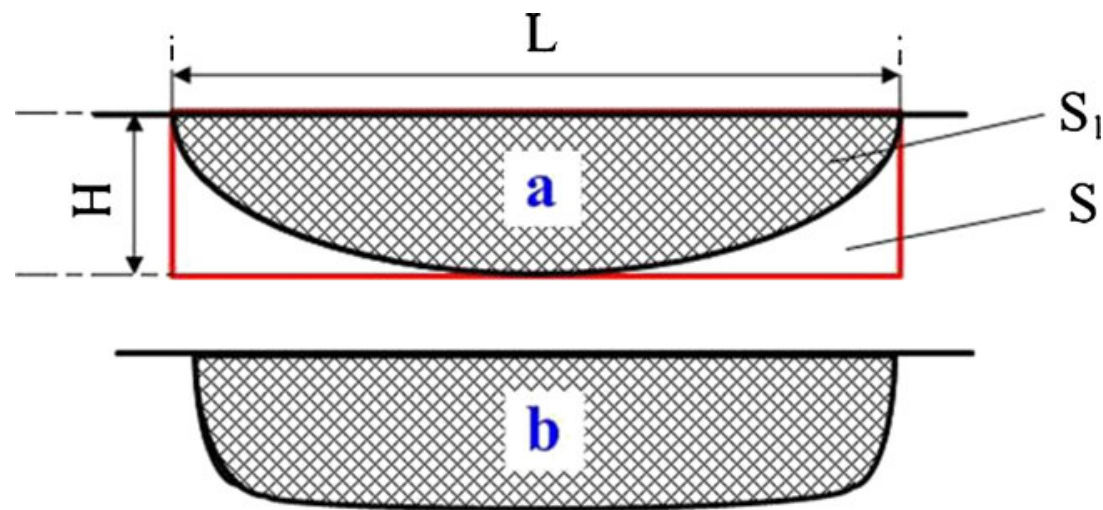


Fig. 14 Cross-section of the hardened layers ( $700 \mathrm{~W} ; 5,10$, 15 , and $20 \mathrm{~mm} / \mathrm{s}$ ) by the Gaussian beam (a), shaped beam 1 (b), and shaped beam $2(\mathbf{c})$
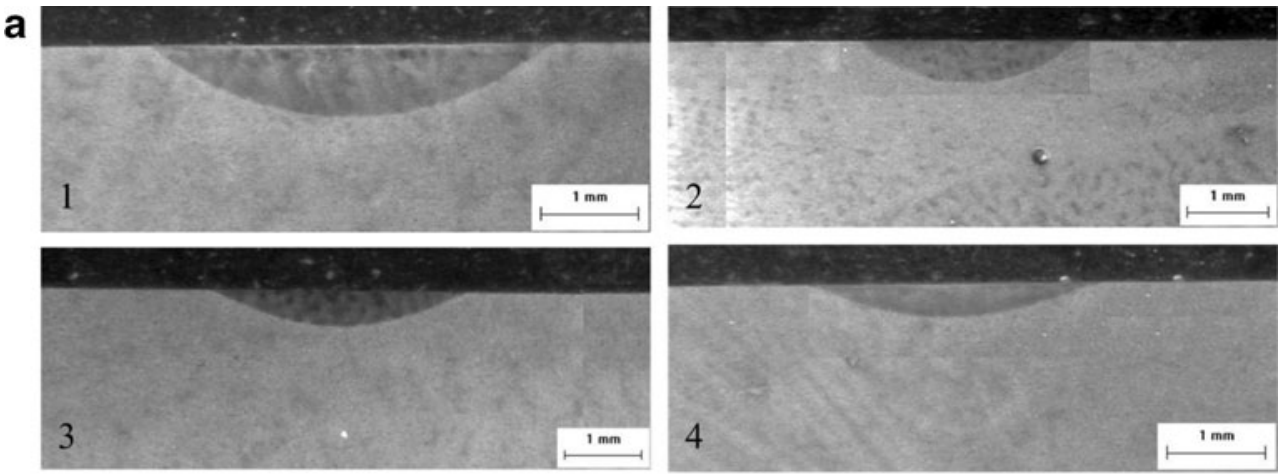

b
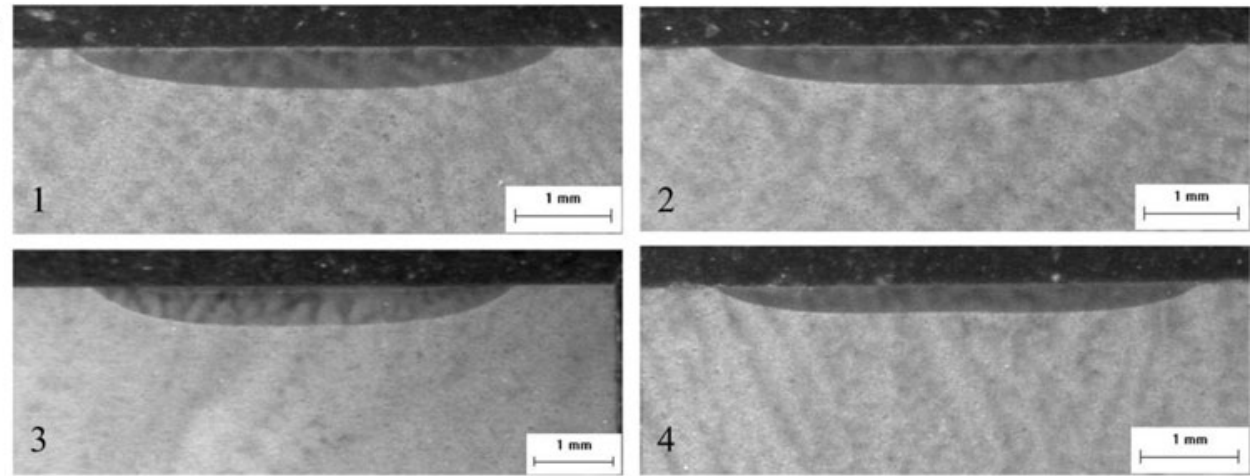

C
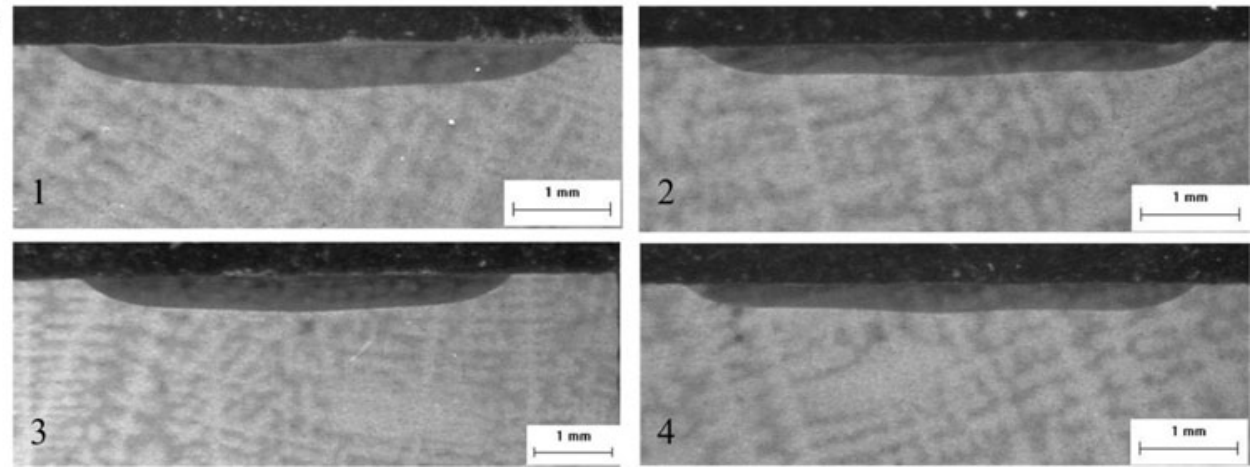

where $S_{1}$ denotes the area of the hardened layer profile, $S$ denotes the area of the rectangle which exactly contains the hardened layer profile, $L$ denotes the width of the hardened layer, and $H$ denotes the depth of the hardened layer. The
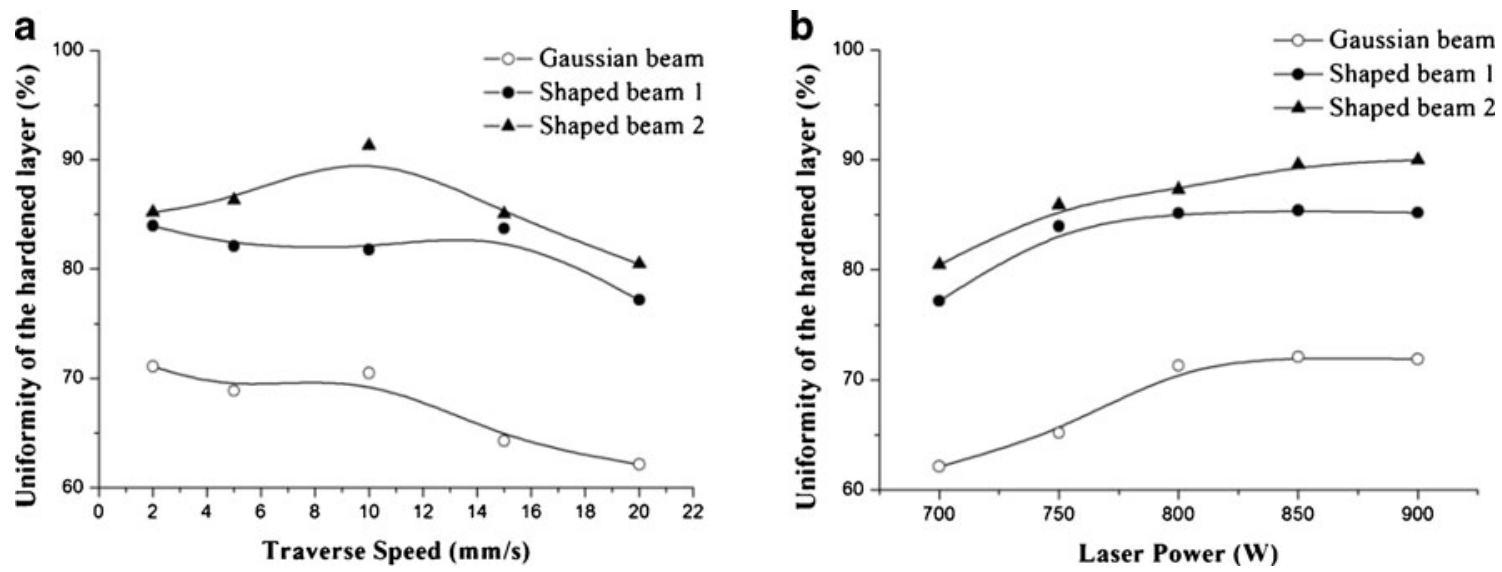

Fig. 15 Uniformity of the hardened layers under different scanning velocities (a) and laser powers (b) 
uniformity coefficient $R$ actually means the geometrical uniformity of the hardened layer. It can be seen that the larger the uniformity coefficient $R$, the more uniform is the hardened layer.

The morphology of the cross-section of the hardened layers by the three beams is shown in Fig. 14. The laser power used was $700 \mathrm{~W}$; the scanning velocities used were $5,10,15$, and $20 \mathrm{~mm} / \mathrm{s}$, which correspond to $1,2,3$, and 4 in Fig. 14. According to the definition of uniformity of the hardened layer, values of $R$ are in the range of $62-72 \%$ in the case of the Gaussian beam (Fig. 14a) and are in the range of 77-85\% in the case of shaped beam 1 (Fig. 14b); in the case of shaped beam 2 , the values are from 82 to $91 \%$ (Fig. 14c). Values of $R$ in the case of shaped beam 1 are higher than those in the case of Gaussian beam; the reason is that this beam shape has a higher energy efficiency along the width of hardened layer, and the reason why values of $R$ in the case of shaped beam 2 are higher than those in the case of shaped beam 1 is that more energy is supplied in the edge of shaped beam 2. It reveals that laser beam geometry and intensity distribution have an important influence on the uniformity of the hardened layer.

Uniformity of the hardened layers under the Gaussian beam and shaped beams 1 and 2 is shown and compared in Fig. 15a. Figure 15b shows the values of $R$ under different laser powers (laser scanning velocity, $20 \mathrm{~mm} / \mathrm{s}$ ). As illustrated in Fig. 15a, the variety of $R$ seems to have no rules with scanning velocity increasing. It can be seen from Fig. $15 \mathrm{~b}$ that a plateau exists with laser power increasing, which means that the value of $R$ cannot increase unlimitedly. When the laser power is high enough, uniformity of the hardened layer will approach a fixed value.

\section{Conclusion}

The process of laser surface hardening of $42 \mathrm{CrMo}$ cast steel by shaped beams has been analyzed. A three-dimensional FEM has been developed to calculate the temperature history of special points to help in understanding the microstructure and microhardness achieved. The main conclusions are summarized as follows:

1. Due to the large length-to-width ratio for shaped beams, the widths of the hardened layers by these beams were much larger than that of the Gaussian laser beam. The shaped beam was more suitable for relatively higher laser scanning velocities to get a wider and no shallow hardened layer.

2. Laser beam geometry and intensity distribution affect the uniformity of the hardened layer significantly. The uniformity of the hardened layers by shaped beam 2 was better than the other two beams due to its beam shape and intensity blowup in the edge. But the uniformity of the hardened layers cannot increase unlimitedly, and when the laser power is high enough, the uniformity coefficient will approach a fixed value.

3. The thermal history of a material has an important effect on the microstructure and microhardness finally formed. Shaped beam has a higher hardness in the transformation zone due to the high heating rate and peak temperature.

Acknowledgments This work was supported by the National Natural Science Foundation of China under grant no. 10832011.

\section{References}

1. Shin HJ, Yoo YT, Ahn DG, Im K (2007) Laser surface hardening of S45C medium carbon steel using ND:YAG laser with a continuous wave. J Mater Process Technol 187-188:467-470

2. Wu W, Liang NG, Gan CH, Yu G (2006) Numerical investigation on laser transformation hardening with different temporal pulse shapes. Surf Coat Technol 8:2686-2694

3. Dinesh Babu P, Buvanashekaran G, Balasubramanian KR (2013) Experimental investigation of laser transformation hardening of low alloy steel using response surface methodology. Int J Adv Manuf Technol 67:1883-1897

4. Xu H, Chen WW, Zhou K, Huang Y, Wang QJ (2010) Temperature field computation for a rotating cylindrical workpiece under laser quenching. Int J Adv Manuf Technol 47:679-686

5. Giorleo L, Previtali B, Semeraro Q (2011) Modelling of back tempering in laser hardening. Int J Adv Manuf Technol 54:969-977

6. Chen SL, Shen D (1999) Optimization and quantitative evaluation of the qualities for Nd-YAG laser transformation hardening. Int $\mathrm{J}$ Adv Manuf Technol 15:70-78

7. Zhou P, Ma YX, Wang XL, Ma HT, Xu XJ, Liu ZJ (2009) Average intensity of a partially coherent rectangular flat-topped laser array propagating in a turbulent atmosphere. Appl Optics 48:5251-5258

8. Li CW, Wang Y, Han B (2011) Microstructure, hardness and stress in melted zone of $42 \mathrm{CrMo}$ steel by wide-band laser surface melting. Opt Lasers Eng 49:530-535

9. Hagino H, Shimizu S, Ando H, Kikuta H (2010) Design of a computer-generated hologram for obtaining a uniform hardened profile by laser transformation hardening with a high-power diode laser. Precis Eng 34:446-452

10. Hwang J-H, Lee Y-S, Kim D-Y, Youn J-G (2002) Laser surface hardening of gray cast iron used for piston ring. J Mater Eng Perform 11:294-300

11. Pantelis D, Bouyiouri E, Kouloumbi N, Vassiliou P, Koutsomichalis A (2002) Wear and corrosion resistance of laser surface hardened structural steel. Surf Coat Technol 161:125-134

12. Sandven O (1981) Laser surface transformation hardening. Metals handbook, 9th edn. ASM, Metals Park, $\mathrm{OH}$

13. Soriano C, Leunda J, Lambarri J, Garcia Navas V, Scanz C (2011) Effect of laser hardening on the microstructure, hardness and residual stresses of austempered ductile iron grades. Appl Surf Sci 257:71017106

14. Leung MKH, Man HC, Yu JK (2007) Theoretical and experimental studies on laser transformation hardening of steel by customized beam. Int J Heat Mass Transfer 50:4600-4606

15. Kock DJ (2004) Lasers offer unique heat treating capabilities. www.industrialheating.com

16. Safdar S, Li L, Sheikh MA, Liu Z (2006) An analysis of the effect of laser beam geometry on laser transformation hardening. J Manuf Sci E-T ASME 12:8659-8667 